\title{
CMEARTICLE \\ Clinics in diagnostic imaging (152)
}

Jerome Irai Ezhil Bosco ${ }^{1}$, MBBS, MD, Ree Nee $\underline{K h o O}^{1}$, MMed, FRCR, Wilfred CG $\underline{\operatorname{Peh}}^{1}$, FRCP, FRCR
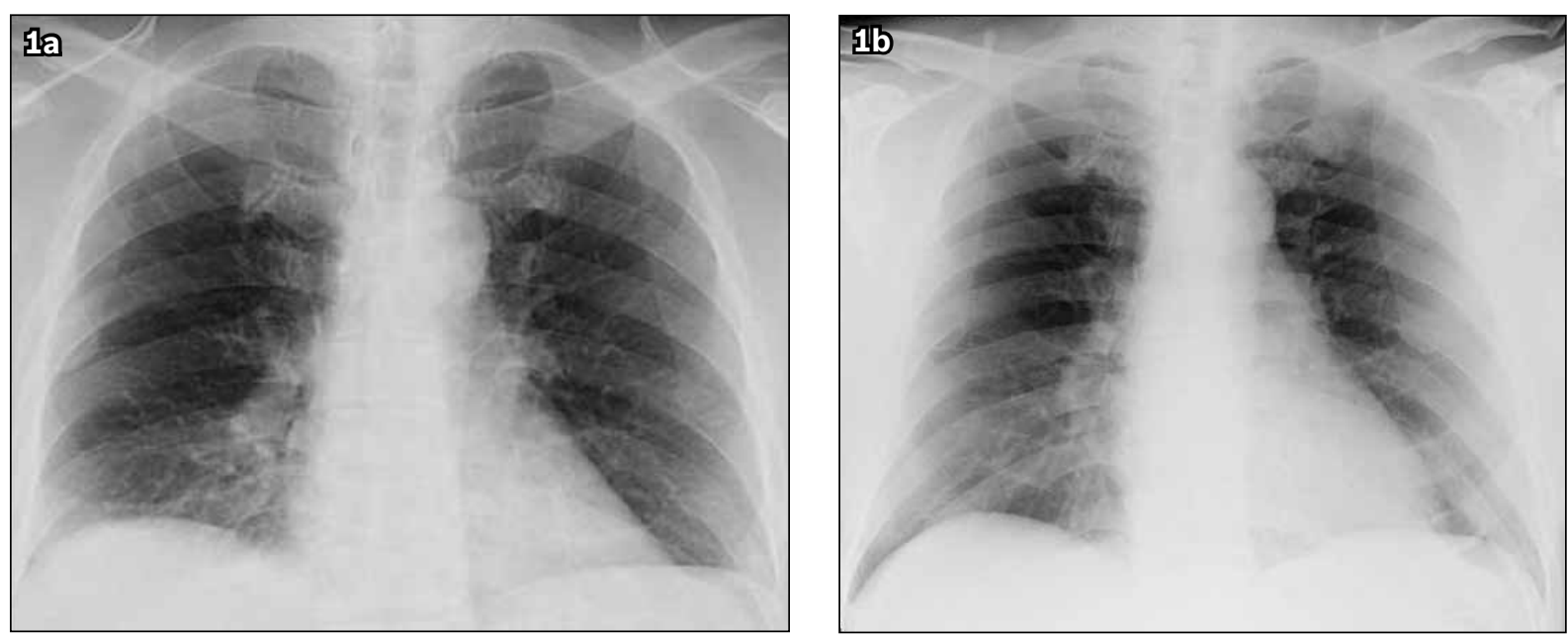

Fig. 1 Chest radiographs of the patient taken (a) at the time of presentation of acute chest pain and (b) three months earlier.
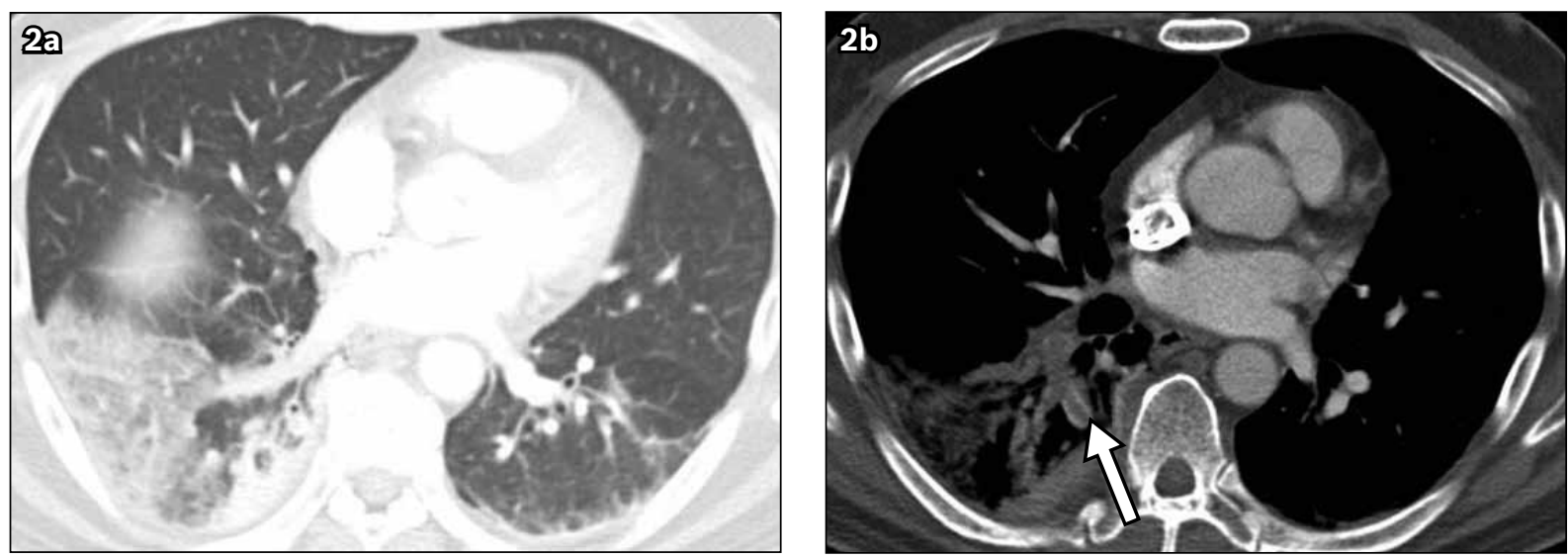

Fig. 2 Axial CTPA images of the same patient in (a) lung window and (b) mediastinal window taken at the level of the inferior pulmonary veins.

\section{CASE PRESENTATION}

A 56-year-old man presented to the Accident and Emergency Department with severe right-sided pleuritic chest pain of sudden onset since the previous night. There was exacerbation of pain on movement, with associated shortness of breath, and a mild fever. He had a history of air travel of five hours' duration ten days prior to presentation. A chest radiograph (Fig. 1a) was done, followed by computed tomography pulmonary angiography (CTPA) (Fig. 2). The plasma D-dimer level was raised at $5.28 \mathrm{mg} / \mathrm{L}$ fibrinogen equivalent units (normal $<0.55 \mathrm{mg} / \mathrm{L}$ ). Comparison was made with a chest radiograph (Fig. 1b) taken three months ago. What are the findings? What is the diagnosis?

${ }^{1}$ Department of Diagnostic Radiology, Khoo Teck Puat Hospital, Singapore

Correspondence: Prof Wilfred CG Peh, Professor and Head, Department of Diagnostic Radiology, Khoo Teck Puat Hospital, Alexandra Health, 90 Yishun Central, Singapore 768828.wilfred.peh@gmail.com 

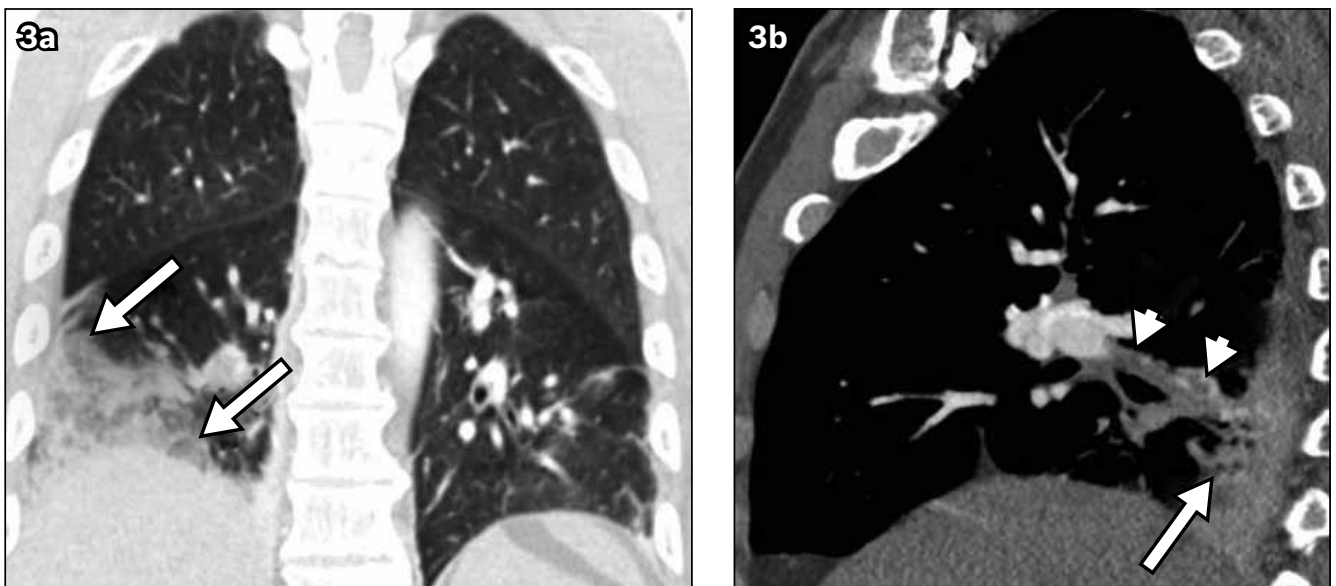

Fig. 3 (a) Coronal CTPA image in lung window shows the peripherally located infarcted lung (arrows), corresponding to the Hampton's hump sign seen on the chest radiograph. (b) Sagittal CTPA image in mediastinal window shows thrombi within the right lower lobe segmental branches (arrowheads) and the peripherally located infarcted lung (arrow).

\section{IMAGE INTERPRETATION}

Compared with the previous normal chest radiograph (Fig. 1b), the present chest radiograph (Fig. 1a) shows a new pleural-based, wedge-shaped opacity in the right lower zone, with a broad base toward the pleura and its apex toward the hilum. This finding is known as Hampton's hump. The adjoining medial lung field is oligaemic, consistent with the Westermark sign. The ipsilateral right descending pulmonary artery appears to be mildly dilated; this is called Palla's sign. CTPA shows a focus of collapseconsolidation at the posterior segment of the right lower lobe (Fig. 2a) with thrombosis within the right lower lobe segmental arteries (arrow; Fig. 2b). These findings are further defined on reconstructed coronal and sagittal CTPA images obtained in lung and mediastinal windows (Figs. 3a \& b, respectively).

\section{DIAGNOSIS}

Right lower lobe segmental pulmonary embolus.

\section{CLINICAL COURSE}

The patient was started on subcutaneous Clexane (lowmolecular-weight heparin, enoxaparin sodium), followed by oral warfarin, with close monitoring of his prothrombin time and international normalised ratio (INR). He showed significant symptomatic improvement with anticoagulation treatment and was discharged after a one-week stay in the hospital. A review of his past medical history revealed an earlier diagnosis of nephrotic syndrome. A previous renal biopsy showed membranous glomerulopathy, which is a predisposing factor to venous thromboembolism (VTE). ${ }^{(1)}$ Colour Doppler ultrasonography of his lower limbs was, however, negative for deep vein thrombosis. At the last follow-up in the outpatient clinic, the patient was asymptomatic and advised to continue with prophylactic oral warfarin.

\section{DISCUSSION}

Pulmonary embolism (PE) is the third most common acute cardiovascular disease (after myocardial infarction and stroke) and the most common cause of death after elective surgery (accounting for up to $15 \%$ of all postoperative deaths), with an untreated mortality of about $30 \%$. It is also the most common cause of maternal death in some countries - in the United Kingdom, the mortality rate was 1.56 per 100,000 pregnancies. ${ }^{(2,3)}$ In the general population, mortality depends on the severity of $\mathrm{PE}$, with the risk of dying within 90 days occurring in up to $50 \%$ of patients with massive PE (associated systemic arterial hypotension).(3) The annual incidence of this life-threatening condition is about $60-70$ per $100,000 .{ }^{(4)}$

The most common cause of PE is thrombi generated in the deep venous system of the lower leg and pelvis, which is facilitated by venous stasis, with some individuals showing a genetic predisposition for this process. Up to $50 \%$ of venous thrombi in the lower limb eventually embolise, with those above the knee being more common. ${ }^{(2,5)}$ Large clots may lodge at the bifurcation of the main pulmonary arteries, causing haemodynamic compromise, while smaller clots travel more distally, resulting in lung infarction and pleuritic pain. The major risk factors for PE include obesity, cigarette smoking, hypertension, prolonged immobilisation (due to major surgery), late pregnancy, oral contraceptives, trauma, thrombophilia (including nephritic syndrome) and malignancy. ${ }^{(2)} \mathrm{A}$ less common cause that has captured public imagination is the 'economy class syndrome' or 'travellers' thrombosis' seen in long distance sedentary air travel, with an incidence of VTE of about 3.2 per 1,000 person-years. ${ }^{(6)}$ This translates to an incidence of PE of 1.65 per million patients in flights that last longer than 8 hours and up to 4.8 per million patients in flights longer than 12 hours or distances exceeding $10,000 \mathrm{~km}{ }^{(6)}$ Although the absolute risk of VTE is low, it is still about thrice as high when compared to the normal, healthy 'non-flying' population, and is significant because of the volume of air travel in recent times. Some of the important guidelines advocated by the American College of Chest Physicians and Aerospace Medical Association for healthy travellers taking flights of more than 8 hours' duration include avoidance of tight clothing for the lower body, maintenance of adequate hydration, and frequent exercise of the legs..$^{(7)}$ 

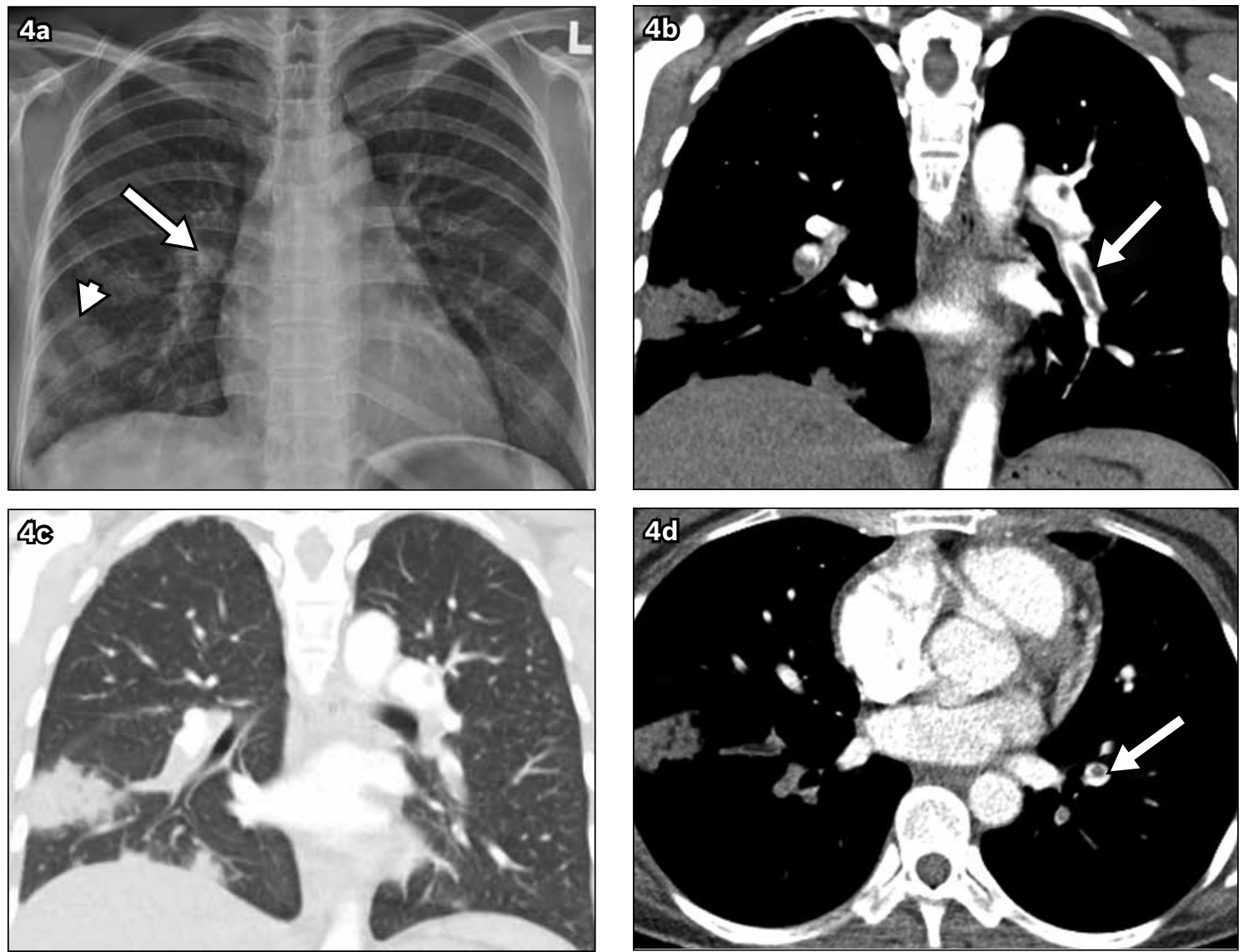

Fig. 4 A 34-year-old woman with known antiphospholipid syndrome presented with right-sided chest pain and haemoptysis. (a) Chest radiograph shows a right lower zone peripheral opacity representing a Hampton's hump (arrowhead). Palla's sign (arrow) is also present. (b) Coronal CTPA image (mediastinal window) shows a thrombus in the right lower lobe segmental artery, enlarging its lumen, with pleuralbased lung infarct. The left lower lobe segmental artery shows the characteristic 'tram-track' sign (arrow) due to the thrombus being surrounded by the contrast-filled artery lumen. (c) Coronal CTPA image (lung window) taken at the same level as the image in Fig. $4 \mathrm{~b}$ better shows the right pleural-based lung infarct. (d) Axial CTPA image (mediastinal window) shows thrombi within the lower lobe segmental branches bilaterally, and the right pleural-based lung infarct. The left lower lobe segmental branch shows the characteristic 'polo-mint' sign (arrow).
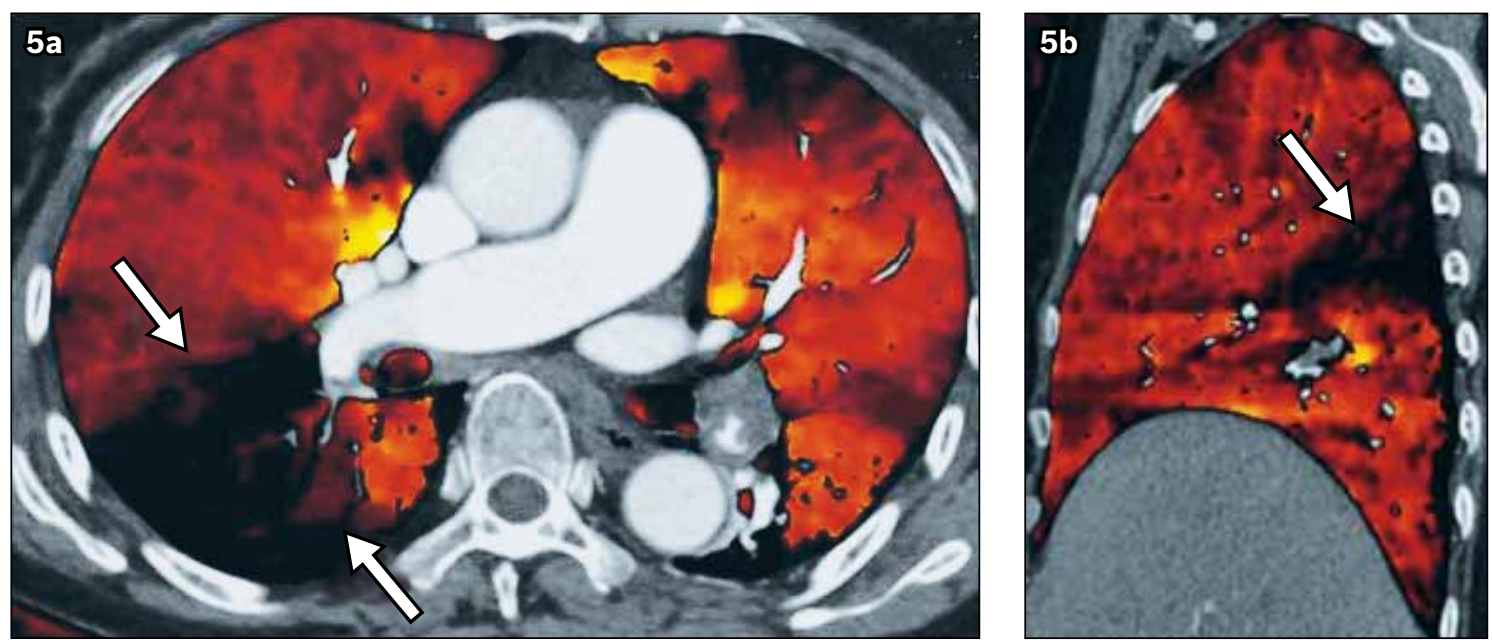

Fig. 5 (a) Axial and (b) sagittal vascular iodine distribution maps (a recent innovation of dual-energy CT for showing lung perfusion) in a 69-yearold woman who presented with chest pain that was worse on exertion. These images are colour-coded as per choice, and a characteristic dark wedge-shaped defect (arrows) due to pulmonary embolism is seen in the apical segment of the right lower lobe. The rest of the lung shows normal perfusion.

The most common presenting symptoms of PE are dyspnoea and tachypnoea (respiratory rate $>20 / \mathrm{min}$ ). Many pretest clinical probability scores are used in the assessment of the clinical likelihood of $\mathrm{PE}$, a popular one being the British Thoracic Society guidelines for the management of suspected acute pulmonary embolism. ${ }^{(8)}$ Plasma D-dimer, a degradation product of cross-linked fibrin (normal value $<0.55 \mathrm{mg} / \mathrm{L}$ fibrinogen equivalent units), is a useful tool for diagnosing PE; however, most studies show that plasma D-dimer is sensitive (up to $94 \%$ ) but not very specific (about $45 \%$ ). A negative test 


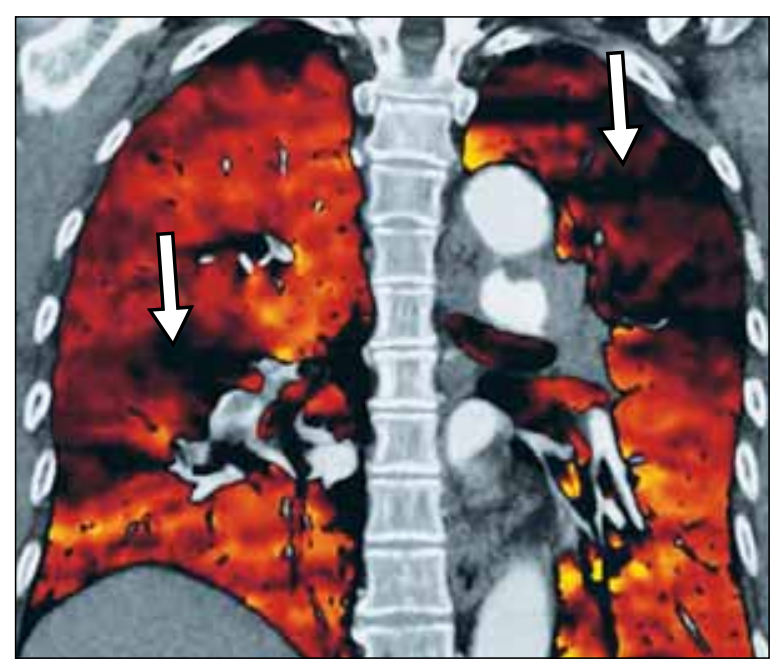

Fig. 6 Coronal CT vascular iodine distribution map of a 31-year-old woman who presented with right calf pain and dry cough. Dark wedgeshaped perfusion defects (arrows) in the right lower and left upper lungs, typical of bilateral pulmonary embolism, are seen.

result reliably excludes PE in patients with low pretest clinical probability. ${ }^{(9)}$

Autopsy studies reveal that up to $5 \%$ of deaths attributed to PE were missed during life. ${ }^{(10)}$ However, with innovations in CT imaging technology and the introduction of multidetector CTPA (now considered the diagnostic gold standard), the detection rate of PE has significantly improved, with a sensitivity of $83 \%$ and a specificity of up to $100 \%{ }^{(11,12)}$ The prospective investigation of pulmonary embolism diagnosis (PIOPED) II investigators and British Thoracic Society suggest that it should be the first line of investigation in all patients with a high clinical probability of $\mathrm{PE} .{ }^{(9)}$ The characteristic CT finding in acute PE is a soft tissue density filling defect partly or entirely filling the lumen, sometimes enlarging it. A partial thrombus shows an acute angle to the arterial wall, with contrast filling the patent lumen, giving rise to the 'polo-mint' sign when seen en face, or the 'tram-track' sign when seen at right angles to the vessel. The lung infarct is seen as a peripheral wedge-shaped area or areas of hyperattenuation (Fig. 4). CTPA findings of right ventricular dilatation, deviation of the interventricular septum toward the left, contrast material reflux into the hepatic veins, and pulmonary embolism index greater than $60 \%$ give valuable information about right heart strain (a common complication of PE) and its extent. Chronic PE findings on CTPA include complete occlusion of an artery by a thrombus with vessel calibre smaller than normal, a peripheral crescent-shaped intraluminal defect that forms an obtuse angle with the vessel wall, contrast material flowing through thickened and often smaller arteries due to recanalisation, or a web/flap within a contrast-filled artery. Indirect signs of chronic PE include bronchial/systemic collaterals, arterial wall calcification, enlarged main pulmonary artery and post-stenotic dilatation. Lungs in chronic PE show a characteristic mosaic pattern, due to nonuniform arterial perfusion. ${ }^{(12)} \mathrm{A}$ big advantage of CTPA is that it helps rule out the differentials of PE with acute onset

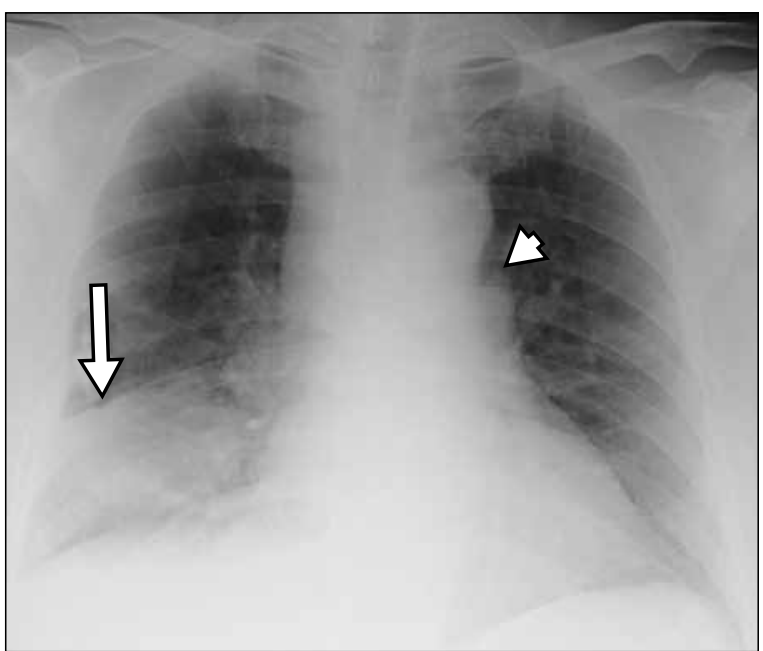

Fig. 7 Chest radiograph of a 61-year-old man, a known smoker, who presented with right-sided chest pain for a day. Right lower zone pleural-based opacity, representing a Hampton's hump (arrow), is seen. Prominent main pulmonary artery is seen as convexity of the pulmonary bay, typical of Fleischner's sign (arrowhead).

chest pain, notably aortic aneurysm/dissection and acute coronary syndrome. It is not surprising that multidetector CT protocols in accident and emergency for 'triple scans' to rule out the 'big three' chest emergencies are being actively promoted.

Multidetector CTPA technology has stretched the threshold of isotropic imaging and visual acuity, with submillimetre reconstruction allowing the detection of the smallest visible thrombi. The most recent innovation in CT technology is the use of dual-energy, which gives functional information, notably lung perfusion using vascular iodine distribution maps ${ }^{(13)}$ (see Figs. 5 \& 6). Characteristic wedge-shaped perfusion defects are seen in PE, and the volume of infarcted lung thus calculated is seen to have good correlation with the clinical outcome. ${ }^{(14)}$ It has been shown that the use of vascular iodine maps increases the sensitivity of detecting PE when used in conjunction with CTPA $^{(15)}$ (see Figs. 5 \& 6). Recent studies show that dual-energy CTPA involves lower radiation doses and smaller amounts of intravenous contrast than the equivalent single-energy examination. ${ }^{(16)}$ However, there is some controversy regarding the overuse of technology and overtreatment of $\mathrm{PE}$, especially with detection of isolated subsegmental pulmonary arterial thrombi on multidetector CTPA. ${ }^{(17)}$ Magnetic resonance (MR) angiography (especially with the newer time-resolved sequences) with added functional information of pulmonary perfusion imaging has shown promising results in the detection of $\mathrm{PE}$, and is the modality to look out for in the future. ${ }^{(18)}$

In the past, ventilation-perfusion (V/Q) lung imaging played an important role in diagnosing $\mathrm{PE}$, but critical reviews regarding its clinical usefulness and the rapid advances in multidetector CT technology have relegated it to a secondary role. At present, V/Q imaging is only indicated in PE when a patient has renal failure or a severe iodinated contrast allergy. Recent studies give V/Q imaging a very decent sensitivity of $77.4 \%$ and a specificity of $97.7 \%$ in the detection of $\mathrm{PE}$, which is comparable 
to CTPA. ${ }^{(19)}$ Radiation exposure using CTPA and V/Q imaging are also comparable, with effective whole body dosages of 1.6-8.3 mSv and 1.2-2.0 mSv, respectively. ${ }^{(9)}$

Chest radiograph remains the first investigation conducted when a patient presents with the classical clinical triad of PE - namely chest pain, shortness of breath and hypoxia. A few uncommon but characteristic radiographic findings such as Hampton's hump, Westermark sign and Fleischner's sign have been documented (Figs. 1, 4 \& 7). Hampton's hump is a pleuralbased consolidation, partly wedge-shaped with a rounded convex apex directed toward the hilum ${ }^{(20)}$ that appears within two days of a pulmonary infarction. It occurs due to alveolar wall necrosis and alveolar haemorrhage within an incomplete infarct, which gives a more rounded appearance of a 'hump', as opposed to a pure wedge shape. First described by Aubrey Otis Hampton in 1940, this sign is commonly encountered at the lower lobes (although this depends on the site of infarction) and slowly resolves over several months, usually with a residual scar - a process described as the 'melting sign'. Healing by cavitation has also been described. An increased translucency (oligaemic lung fields) on frontal radiographs is named Westermark sign, which occurs due to a decrease in vascularisation at the periphery of the lungs by primary mechanical obstruction or reflex vasoconstriction. ${ }^{(21)}$ Fleischner's $\operatorname{sign}^{(22)}$ describes a prominent central pulmonary artery. Knuckle $\operatorname{sign}^{(22)}$ refers to an abrupt tapering of an occluded pulmonary artery, while Palla's sign ${ }^{(23)}$ is an enlargement of the right descending pulmonary artery. Other radiographic findings in PE include atelectasis, infiltrates, an elevated diaphragm and pleural effusion. Interestingly, Elliott et al found that the most common finding in PE on radiographs was cardiomegaly in patients older than 70 years, while a normal radiograph was most common in those younger than 70 years. ${ }^{(24)}$

Treatment for PE comprises anticoagulation medication, with heparin (low-molecular-weight heparin, unfractionated heparin) and vitamin K antagonists (warfarin) used in haemodynamically stable patients, while thrombolysis is required in haemodynamically unstable patients. Inferior vena cava filters can be inserted under image guidance whenever anticoagulation is contraindicated or unsuccessful in preventing recurrence of PE from continuing DVT.

\section{REFERENCES}

1. Blann AD, Lip GY. Venous thromboembolism. BMJ 2006; 332:215-9.

2. Robinson GV. Pulmonary embolism in hospital practice. BMJ 2006; 332:156-60.

3. Kucher N, Rossi E, De Rosa M, and Goldhaber SZ. Massive pulmonary embolism. Circulation 2006; 113:577-82.

4. Moser KM, Fedullo PF, LittleJohn JK, Crawford R. Frequent asymptomatic pulmonary embolism in patients with deep venous thrombosis. JAMA 1994; 271:223-5.

5. Goldhaber SZ, Grodstein F, Stampfer MJ, et al. A prospective study of risk factors for pulmonary embolism in women. JAMA 1997; 277:642-5.

6. Bartholomew JR, Schaffer JL, McCormick GF. Air travel and venous thromboembolism: minimizing the risk. Cleve Clin J Med 2011; 78:111-20.

7. Geerts WH, Bergqvist D, Pineo GF, et al. Prevention of venous
ABSTRACT A 56-year-old man presented to the Accident and Emergency Department with pleuritic chest pain of sudden onset. He gave a history of short-distance air travel ten days earlier. Chest radiograph showed a peripheral-based opacity in the right lower zone, which was not seen in a previous study done three months ago, suggestive of Hampton's hump. The D-dimer level was raised. Computed tomography pulmonary angiography confirmed the diagnosis of pulmonary embolism in a right lower lobe segmental branch, with adjacent collapsed lung, consistent with lung infarction. The patient was started on heparin injection with significant relief of his symptoms. The clinical and imaging features of pulmonary embolism are described, with emphasis on the historical radiographic signs and the current dualenergy computed tomography innovations.

Keywords: computed tomography pulmonary angiography, dual-energy computed tomography, Hampton's hump, pulmonary embolism, venous thromboembolism

thromboembolism: American College of Chest Physicians EvidenceBased Clinical Practice Guidelines. 8th ed. Chest 2008; 133 (Suppl 6):381S-453S

8. British Thoracic Society Standards of Care Committee Pulmonary Embolism Guideline Development Group. British Thoracic Society guidelines for the management of suspected acute pulmonary embolism. Thorax 2003; 58:470-83.

9. Stein PD, Woodard PK, Weg JG, et al. Diagnostic pathways in acute pulmonary embolism: recommendations of the PIOPED II Investigators. Radiology 2007; 242:15-21.

10. Stein PD, Henry JW. Prevalence of acute pulmonary embolism among patients in a general hospital and at autopsy. Chest 1995; 108:978-81.

11. Nazaroglu H, Ozmen CA, Akay HO, Kilinç I, Bilici A. 64-MDCT pulmonary angiography and CT venography in the diagnosis of thromboembolic disease. Am J Roentgenol 2009; 192:654-61.

12. Wittram C, Maher MM, Yoo AJ, et al. CT angiography of pulmonary embolism: diagnostic criteria and causes of misdiagnosis. Radiographics 2004; 24:1219-38.

13. Thieme SF, Johnson TR, Reiser MF, Nikolaou K. Dual-energy lung perfusion computed tomography: a novel pulmonary functional imaging method. Semin Ultrasound CT MR 2010; 31:301-8.

14. Thieme SF, Ashoori N, Bamberg F, et al. Severity assessment of pulmonary embolism using dual energy CT - correlation of a pulmonary perfusion defect score with clinical and morphological parameters of blood oxygenation and right ventricular failure. Eur Radiol 2012; 22:269-78.

15. Zhang LJ, Zhao YE, Wu SY, et al. Pulmonary embolism detection with dualenergy CT: experimental study of dual-source CT in rabbits. Radiology 2009; 252:61-70.

16. Godoy MCB, Heller SL, Naidich DP, et al. Dual-energy MDCT: comparison of pulmonary artery enhancement on dedicated CT pulmonary angiography, routine and low contrast volume studies, Eur J Radiol 2011; 79:e11-7.

17. Suh JM, Cronan JJ, Healey TT. Dots are not clots: the over-diagnosis and over-treatment of PE. Emerg Radiol 2010; 17:347-52.

18. Kluge A, Luboldt W, Bachmann G. Acute pulmonary embolism to the subsegmental level: diagnostic accuracy of three MRI techniques compared with 16-MDCT. AJR Am J Roentgenol 2006; 187:W7-14.

19. Sostman HD, Stein PD, Gottschalk A, et al. Acute pulmonary embolism: sensitivity and specificity of ventilation-perfusion scintigraphy in PIOPED II study. Radiology 2008; 246:941-6.

20. Hampton AO, Castleman B. Correlation of postmortem chest teleroentgenograms with autopsy findings with special reference to pulmonary embolism and infarction. Am J Roentgenol Radium Ther 1940; 43:305-26.

21. Westermark N. On the roentgen diagnosis of lung embolism. Acta Radiol 1938; 19:357-72.

22. Ho M, Gutierrez F. Chest radiography in thoracic polytrauma. AJR Am J Roentgenol 2009; 192:599-612.

23. Palla A, Donnamaria V, Petruzzelli S, et al. Enlargement of the right descending pulmonary artery in pulmonary embolism. Am J Roentgenol 1983; 141:513-7.

24. Elliott CG, Goldhaber SZ, Visani L, DeRosa M. Chest radiographs in acute pulmonary embolism: results from the International Cooperative Pulmonary Embolism Registry. Chest 2000; 118:33-8. 


\section{SINGAPORE MEDICAL COUNCIL CATEGORY 3B CME PROGRAMME} (Code SMJ 201405B)

Question 1. Regarding the epidemiology and aetiology of pulmonary embolism (PE):

(a) It is the most common cause of postoperative death in elective surgery.

(b) At autopsy, up to 5\% of cases attributed to PE are known to have been missed during life.

(c) Large clots in the main pulmonary arteries cause haemodynamic compromise.

(d) Up to $50 \%$ of thrombi in the lower limbs do eventually embolise.

Question 2. Regarding the clinical features and risk factors in PE:

(a) The most common presenting complaints are dyspnoea and tachypnoea.

(b) Contraception in women is a risk factor.

(c) It is seen in up to $25 \%$ of patients who take long distance flights.

(d) Central venous line thrombosis is the most common cause of PE.

Question 3. Regarding chest radiography findings in PE:

(a) In patients with PE who are younger than 70 years, the most common finding is cardiomegaly.

(b) Hampton's hump is a wedge-shaped pleural-based opacity with its apex toward the hilum.

(c) The Westermark sign refers to increased translucency in the peripheral lung due to resulting vasoconstriction.

(d) An oligaemic lung field peripheral to the site of occlusion is called Fleischner's sign.

Question 4. Regarding the management of PE:

(a) A negative D-dimer test reliably excludes PE in a patient with high clinical probability.

(b) Thrombolysis may be needed for haemodynamically unstable patients.

(c) Inferior vena cava filters are indicated when anticoagulation fails to prevent recurrence of PE.

(d) Multidetector computed tomography (MDCT) pulmonary angiography is considered the gold standard for the detection of PE.

Question 5. Regarding MDCT imaging in PE:

(a) Right ventricle dilatation with bowing of the interventricular septum is an indirect sign of PE.

(b) The smallest thrombi visible to the naked eye can be detected on MDCT.

(c) Dual-energy CT applications include lung perfusion analysis.

(d) Dual-energy CT involves more radiation than single-energy CT imaging.

$\begin{array}{cc}\text { True } & \text { False } \\ \square & \square \\ \square & \square \\ \square & \square \\ \square & \square \\ & \\ \square & \square \\ \square & \square \\ \square & \square \\ \square & \square \\ & \\ \square & \square \\ \square & \square \\ \square & \square \\ \square & \square \\ & \\ \square & \square \\ \square & \square \\ \square & \square \\ \square & \square \\ & \\ \square & \square \\ \square & \square \\ \square & \square \\ \square & \square \\ \square & \square \\ & \square\end{array}$

\footnotetext{
Doctor's particulars:

Name in full

MCR number

Specialty:

Email address

(1) Log on at the SMJ website: http://www.sma.org.sg/publications/smjcurrentissue.aspx and select the appropriate set of questions. (2) Provide your name, email address and MCR number. (3) Select your answers and click "Submit".

RESULTS:

(1) Answers will be published in the SMJ July 2014 issue. (2) The MCR numbers of successful candidates will be posted online at the SMJ website by 24 June 2014. (3) Passing mark is $60 \%$. No mark will be deducted for incorrect answers. (4) The SMJ editorial office will submit the list of successful candidates to the Singapore Medical Council. (5) One CME point is awarded for successful candidates.

Deadline for submission: (May 2014 SMJ 3B CME Programme): 12 noon, 17 June 2014.
} 